\title{
Three X-ray transients in M 31 observed with Swift (Research Note)
}

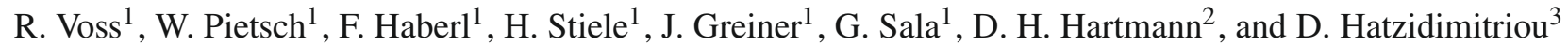 \\ 1 Max-Planck-Institut für extraterrestrische Physik, Giessenbachstrasse, 85748 Garching, Germany \\ e-mail: rvoss@mpe.mpg.de \\ 2 Department of Physics and Astronomy, Clemson University, Clemson, SC 29634, USA \\ 3 University of Crete, Department of Physics, PO Box 2208, 71003 Heraklion, Greece
}

Received 31 October 2007 / Accepted 21 July 2008

\section{ABSTRACT}

\begin{abstract}
Aims. The purpose of this study is to find transient X-ray sources in M 31 and to investigate and classify their nature.
Methods. Three X-ray transients were observed with Swift. For each of the three X-ray transients, we used the Swift X-ray and optical data with observations from XMM-Newton and Chandra to investigate the lightcurves and the spectra of the outburst, thereby identifying the source types.

Results. The outburst of XMMU J004215.8+411924 lasted about one month. The source had a hard power-law spectrum with a photon index of 1.6. It was previously identified as a Be/X-ray binary based on the optical identification with a star. However, we show that, with improved source coordinates it is clear that the optical source is not the counterpart to the X-ray source. The source SWIFT J004217.3+411532 had a bright outburst, after which it slowly decayed over half a year. The spectrum was soft, corresponding to a thermal accretion disc with an innermost temperature of $\sim 250-600 \mathrm{eV}$. The source was not seen in the optical, and the soft spectrum indicates that the source is most likely a black-hole low-mass X-ray binary. M31N 2006-11a is a nova that was previously observed in the optical. We detected it both in X-rays and UV with Swift $\sim$ half a year after the optical maximum, after which it decayed below the Swift detection threshold within a month. The spectrum of the X-ray transient can be modelled by a black-body with a temperature of $50 \mathrm{eV}$. We use catalogues of X-ray transients in M 31 to estimate their rate, and we find a lower limit of $9 \mathrm{yr}^{-1}$.
\end{abstract}

Key words. galaxies: individual: M 31 - X-rays: binaries - X-rays: galaxies

\section{Introduction}

As the nearest large spiral galaxy, M 31 is an important target for the study of X-ray point sources in galaxies. In many studies, X-ray point sources in M 31 have been investigated and catalogued, using Einstein (Trinchieri \& Fabbiano 1991), ROSAT (Primini et al. 1993; Supper et al. 2001), XMM-Newton (e.g. Trudolyubov et al. 2002; Pietsch et al. 2005), and Chandra (e.g. Kong et al. 2003; Kaaret 2002; Williams et al. 2004, 2006; Voss \& Gilfanov 2007).

An interesting subset of these sources are the transients. These are sources with quiescent luminosities below the detection limit $\left(\sim 10^{35} \mathrm{erg} \mathrm{s}^{-1}\right)$, which display bright outbursts typically lasting from weeks to a few months. From Galactic observations, it is well-known that there are three types of objects that can show such outbursts. Optical studies of soft X-ray transients have shown that they contain an accreting compact object more massive than $3 M_{\odot}$, revealing the presence of black hole accretors (McClintock \& Remillard 2006). In contrast, the harder sources typically show pulsations, indicating that the accreting object is a neutron star (e.g. Tanaka \& Shibazaki 1996; Campana et al. 1998). The third class is the thermal emission from classical novae in optical decline, showing up as supersoft X-ray transients (MacDonald \& Vennes 1991; Starrfield 1989; Pietsch et al. 2007b). Monitoring programmes have shown that 6-12 transients appear per year in the bulge of M 31 (Trudolyubov et al. 2006), and within the timescale of the X-ray observations, only a small fraction of them have been observed to recur.

Here we report on the detection and analysis of three transient X-ray sources in the bulge of M 31, using data from Swift, Chandra, and XMM-Newton.

\section{Data analysis}

A $20 \mathrm{ks}$ XMM-Newton observation on 2006 August 9 as part of the monitoring programme of the X-ray supersoft state of the optical novae in the core of $\mathrm{M} 31$ revealed a new bright transient, designated XMMU J004215.8+411924 (Haberl et al. 2006) ${ }^{1}$. In a 6 ks follow-up target-of-opportunity observation with Swift on 2006 September 1, the source was seen again (Pietsch et al. 2006). A second bright transient was detected in this observation and was designated SWIFT J004217.4+411532. A third source was found in a 19.4 ks Swift observation 2007 June 1 (Pietsch et al. 2007a). We identify this source as optical nova M31N 2006-11a, the optical outburst of which was detected about 190 days earlier ${ }^{2}$.

In Table 1 we list the observations analysed in this study. The distance to M 31 was assumed to be $780 \mathrm{kpc}$, and all

\footnotetext{
1 http://www.mpe.mpg.de/xray/research/normal_galaxies/ index.php

2 http://cfa-www.harvard.edu/iau/CBAT_M31.html\# 2006-11a
} 
Table 1. Observations for the analysis of the three X-ray transients: TR1 (XMMU J004215.8+411924), TR2 (SWIFT J004217.4+411532), and TR3 (M31N 2006-11a).

\begin{tabular}{llcccccc}
\hline \hline Observation & Date & Observatory & Instrument & Obs-ID & TR1 & TR2 & TR3 \\
\hline 1 & 2006 Jun. 05 & Chandra & HRC-I & 7283 & - & - & - \\
2 & 2006 Jul. 02 & XMM & EPIC & 0405320501 & - & - & - \\
3 & 2006 Jul. 31 & Chandra & ACIS-I & 7139 & + & - & - \\
4 & 2006 Aug. 09 & XMM & EPIC & 0405320601 & + & - & - \\
5 & 2006 Sep. 01 & Swift & XRT & 00030802001 & + & + & - \\
6 & 2006 Sep. 11 & Swift & XRT & 00030804001 & - & + & - \\
7 & 2006 Sep. 24 & Chandra & ACIS-I & 7140 & - & + & \\
8 & 2006 Sep. 30 & Chandra & HRC-I & 7284 & - & + & - \\
9 & 2006 Nov. 13 & Chandra & HRC-I & 7285 & - & + & - \\
10 & 2006 Dec. 4 & Chandra & ACIS-I & 7064 & & + & \\
11 & 2006 Dec. 31 & XMM & EPIC & 0405320701 & - & + & - \\
12 & 2007 Jan. 16 & XMM & EPIC & 0405320801 & - & + & - \\
13 & 2007 Mar. 11 & Chandra & HRC-I & 7286 & - & - & - \\
14 & 2007 Jun. 01 & Swift & XRT & 00035336001 & - & - & + \\
15 & 2007 Jul. 12 & Swift & XRT & 00030968001 & - & - & - \\
16 & 2007 Jul. 13 & Swift & XRT & 00030968002 & - & - & - \\
17 & 2007 Jul. 18 & Swift & XRT & 00030968004 & - & - & - \\
\hline
\end{tabular}

Note: $\mathrm{a}+$ signifies that the source was detected in this observation, whereas a - signifies that it was not detected.

fluxes and luminosities are given in the $0.5-5 \mathrm{keV}$ range, except for the nova M31N 2006-11a for which the luminosities are given in the $0.1-1.0 \mathrm{keV}$ range. All statistical errors and upper limits are given with $90 \%$ confidence. The data were reduced using SAS version 7.1.0 for XMM-Newton observations, CIAO version 3.4 for the Chandra observations, and HEAsoft version 6.3 for the Swift observations. The spectra were modelled with Xspec, using the tbabs absorption model with the abundance tables of Wilms et al. (2000) and the photoelectric absorption cross-sections of Balucinska-Church \& McCammon (1992) and Yan et al. (1998). For XMM-Newton, we performed simultaneous fits to the spectra from the pn and the two MOS detectors, except for observations where the source was in or near to detector gaps. All luminosities are given as the intrinsic source luminosities, corrected for absorption.

\section{The sources}

\subsection{XMMU J004215.8+411924}

As is shown in Table 1, this source was active in three X-ray observations (Observations 3 to 5) spanning 40 days. From the non-detections in observations 2 and 6 , the maximum duration of the outburst can be constrained to 79 days. In Table 2 the results of modelling the source spectra with three different models, absorbed power-law, disc black-body, and bremsstrahlung are shown. The luminosities are given as $L_{36}=L / 10^{36} \mathrm{erg} \mathrm{s}^{-1}$, and the neutral hydrogen column is given by $N_{\mathrm{H}, 21}=N_{\mathrm{H}} / 10^{21} \mathrm{~cm}^{-2}$, $\Gamma$ is the photon index of the power-law fits, and the temperatures $T_{\mathrm{in}}$ and $T_{\mathrm{b}}$ of the disc black-body and the bremsstrahlung fits are given in keV. Parameters in parentheses were kept fixed at the assumed values and were not the result of fits to the data. The data can be represented equally well by each of the models, and the obtained luminosities agree within the errors. In the following analysis we use the power-law interpretation, due to the relatively hard spectrum (power-law photon index $\Gamma \simeq 1.6$ ), noting that none of our conclusions are affected by the choice of model. The spectrum obtained from observation 4 is shown in Fig. 1, together with the best-fitting power-law model.

In observation 5 the number of source counts is too low to fit a model to the data, and we used the spectrum from observation 4 to estimate the luminosity of the source, $2.1( \pm 0.7) \times 10^{37} \mathrm{erg} \mathrm{s}^{-1}$, a factor of $\sim 4$ lower than in the previous observations. We used observations 2 and 6 to calculate upper limits on the quiescent luminosity of the source, assuming the spectral shape found in observation 4. These upper limits are given in Table 2 and the lightcurve of the transient is shown in Fig. 2. It can be seen that the source is variable by a factor of more than $\sim 300$, with a peak luminosity of $\sim 10^{38} \mathrm{erg} \mathrm{s}^{-1}$. Galactic binaries often show variability factors of $10^{5}-10^{6}$, but only lower limits on the variability factor of 100-1000 can be found with the sensitivity limits in M 31 .

The source was previously identified as a Be/X-ray binary, based on the identification of an optical counterpart observed with the Swift UVOT (Haberl et al. 2006). With the available Chandra data, the position of the source can be improved significantly. We derived the source position from observation (3), giving $\mathrm{RA}(\mathrm{J} 2000)=00: 42: 16.1, \operatorname{Dec}(\mathrm{J} 2000)=+41: 19: 26.7$, with a $1 \sigma$ error of 0.5 arcsec, including the statistical error on the position, as well as the error on the boresight of the observation, estimated from cross-correlation of the source positions with 2MASS (Skrutskie et al. 2006) sources.

We reinvestigated the correlation between the optical and the $\mathrm{X}$-ray sources, and we furthermore investigated the images from the Local Group Survey (LGS) of Massey et al. (2006). In Fig. 3 we show the region of the source in X-rays and optical. There is an offset between the optical source and the position of the Chandra X-ray source of $\sim 4$ arcsec. This is much larger than the position error, and it shows that the match between the X-ray source and the optical counterpart was coincidental. We therefore conclude that the proposed optical source is not the counterpart to the X-ray source. However, the limiting magnitude of the optical observations is not high enough to rule out a Be companion star. For example the limiting magnitude for this area of the LGS in the $V$-band is $\sim 22$, and with a distance modulus of 24.46 and an extinction of $\sim 0.4$ (Han 1996), Be stars can have magnitudes as faint as 25 (Wegner 2000; Garmany \& Humphreys 1985). On the other hand, the Be/X-ray binaries mostly contain more luminous Be stars, and for example, all known Be/X-ray binaries in the Small Magellanic Cloud (Coe et al. 2005) are luminous enough that they would be observed in the optical if they were at the position XMMU J004215.8+411924. The spectrum and lightcurve of the outburst is consistent with a Be/X-ray 
Table 2. The parameters resulting from the fits to the spectra of XMMU J004215.8+411924 and SWIFT J004217.4+411532.

\begin{tabular}{|c|c|c|c|c|c|c|c|c|c|c|c|c|}
\hline \multirow[b]{2}{*}{ Obs } & \multicolumn{4}{|c|}{ Power-law } & \multicolumn{4}{|c|}{ Disc black-body } & \multicolumn{4}{|c|}{ Bremsstrahlung } \\
\hline & $L_{36}$ & $\Gamma$ & $N_{\mathrm{H}, 21}$ & $\chi_{v}^{2}$ & $L_{36}$ & $T_{\text {in }}$ & $N_{\mathrm{H}, 21}$ & $\chi_{v}^{2}$ & $L_{36}$ & $T_{\mathrm{b}}$ & $N_{\mathrm{H}, 21}$ & $\chi_{v}^{2}$ \\
\hline \multicolumn{13}{|c|}{ XMMU J004215.8+411924 } \\
\hline 2 & $<0.4$ & $(1.57)$ & $(4.2)$ & - & $<3.5$ & (1.92) & (2.2) & - & $<3.8$ & $(12.2)$ & (3.6) & - \\
\hline 3 & $110 \pm 30$ & $1.77 \pm 0.33$ & $6.2 \pm 3.7$ & 0.98 & $86 \pm 44$ & $1.55 \pm 0.35$ & $2.5 \pm 2.3$ & 1.05 & $100 \pm 50$ & $6.5 \pm 4.0$ & $5.2 \pm 2.7$ & 0.98 \\
\hline 4 & $76 \pm 12$ & $1.57 \pm 0.08$ & $4.2 \pm 0.5$ & 0.84 & $67 \pm 11$ & $1.92 \pm 0.14$ & $2.2 \pm 0.3$ & 0.87 & $73 \pm 12$ & $12.2 \pm 4.0$ & $3.6 \pm 0.4$ & 0.80 \\
\hline 5 & $21 \pm 1$ & (1.57) & (4.2) & - & $19 \pm 1$ & (1.92) & (2.2) & - & $20 \pm 1$ & (12.2) & (3.6) & - \\
\hline 6 & $<5.8$ & (1.57) & $(4.2)$ & - & $<5.1$ & (1.92) & $(2.2)$ & - & $<5.6$ & (12.2) & (3.6) & - \\
\hline 7 & $<0.8$ & $(1.57)$ & $(4.2)$ & - & $<0.7$ & $(1.92)$ & $(2.2)$ & - & $<0.6$ & (12.2) & (3.6) & - \\
\hline \multicolumn{13}{|c|}{ SWIFT J004217.4+411532 } \\
\hline 4 & $<2.1$ & (3.5) & $(4.0)$ & - & $<0.9$ & $(0.50)$ & $(0.67)$ & - & $<0.9$ & $(1.0)$ & $(1.5)$ & - \\
\hline 5 & $580 \pm 70$ & $3.12 \pm 0.35$ & $4.4 \pm 1.3$ & 1.35 & $270 \pm 30$ & $0.61 \pm 0.05$ & $(0.67)$ & 1.26 & $350 \pm 70$ & $1.26 \pm 0.26$ & $2.18 \pm 0.91$ & 1.28 \\
\hline 6 & $450 \pm 70$ & $3.55 \pm 0.49$ & $4.0 \pm 1.7$ & 0.99 & $180 \pm 30$ & $0.50 \pm 0.06$ & $(0.67)$ & 0.96 & $230 \pm 70$ & $1.07 \pm 0.27$ & $1.13 \pm 1.13$ & 0.86 \\
\hline 7 & $260 \pm 60$ & $3.47 \pm 0.31$ & $5.1 \pm 1.3$ & 0.87 & $97 \pm 7$ & $0.57 \pm 0.04$ & $(0.67)$ & 0.97 & $130 \pm 20$ & $1.15 \pm 0.11$ & $2.0 \pm 0.9$ & 0.88 \\
\hline 8 & $290 \pm 10$ & $(3.5)$ & $(4.0)$ & - & $140 \pm 10$ & $(0.50)$ & $(0.67)$ & - & $122 \pm 4$ & $(1.0)$ & (1.5) & - \\
\hline 9 & $163 \pm 7$ & (3.5) & $(4.0)$ & - & $80 \pm 4$ & $(0.50)$ & $(0.67)$ & - & $69 \pm 3$ & $(1.0)$ & (1.5) & - \\
\hline 10 & $81 \pm 29$ & $4.50 \pm 0.65$ & $5.1 \pm 2.2$ & 1.26 & $20 \pm 2$ & $0.35 \pm 0.03$ & $(0.67)$ & 1.01 & $28 \pm 15$ & $0.58 \pm 0.12$ & $1.91 \pm 1.33$ & 1.10 \\
\hline 11 & $36 \pm 4$ & $4.34 \pm 0.33$ & $3.3 \pm 0.5$ & 1.56 & $15 \pm 3$ & $0.27 \pm 0.02$ & $(0.67)$ & 1.67 & $18 \pm 4$ & $0.49 \pm 0.06$ & $1.49 \pm 0.27$ & 1.52 \\
\hline 12 & $20 \pm 3$ & $4.51 \pm 0.57$ & $3.2 \pm 0.8$ & 1.10 & $8.2 \pm 2.2$ & $0.25 \pm 0.03$ & (0.67) & 1.44 & $10 \pm 4$ & $0.42 \pm 0.08$ & $1.50 \pm 0.43$ & 1.29 \\
\hline 13 & $<0.9$ & (3.5) & (4.0) & - & $<0.5$ & $(0.50)$ & $(0.67)$ & - & $<0.4$ & (1.0) & (1.5) & - \\
\hline
\end{tabular}

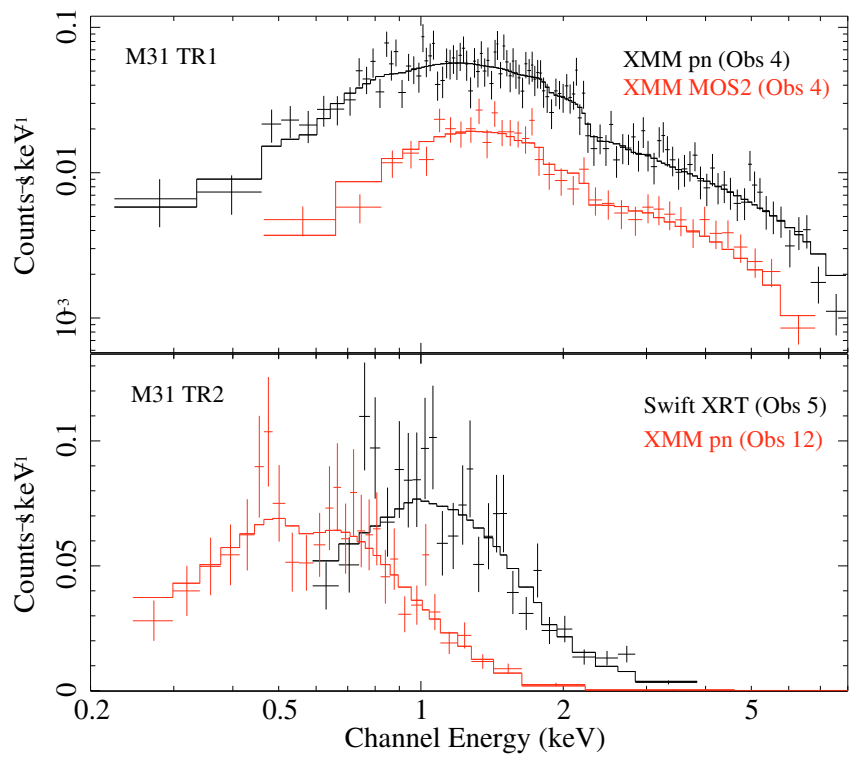

Fig. 1. The spectrum of XMMU J004215.8+411924 (top) from observation 4 , with absorbed power-law fits. The spectrum obtained from the pn (black) and the MOS2 (red) detectors are shown. The source is located in a gap on the MOS1 detector. The spectrum of SWIFT J004217.4+411532 (bottom) from observations 5 (highest observed luminosity, black) and 12 (lowest observed luminosity, red), with absorbed disc black-body fits.

binary in a type II outburst. However, in the absence of a detectable optical counterpart, it is not possible to confirm this.

\subsection{SWIFT J004217.4+411532}

This transient was first observed in the SWIFT follow-up observation of XMMU J004215.8+411924 on 2006 September 1. It stayed in outburst until after January 2007, and it was observed 8 times (observations 5 to 12). The inferred duration of the outburst is between 125 and 175 days. For the observations 5, 6, 11 , and 12 , we extracted source spectra and performed fits using the same three models as for XMMU J004215.8+411924. The results of this analysis are shown in Table 2. The spectrum is

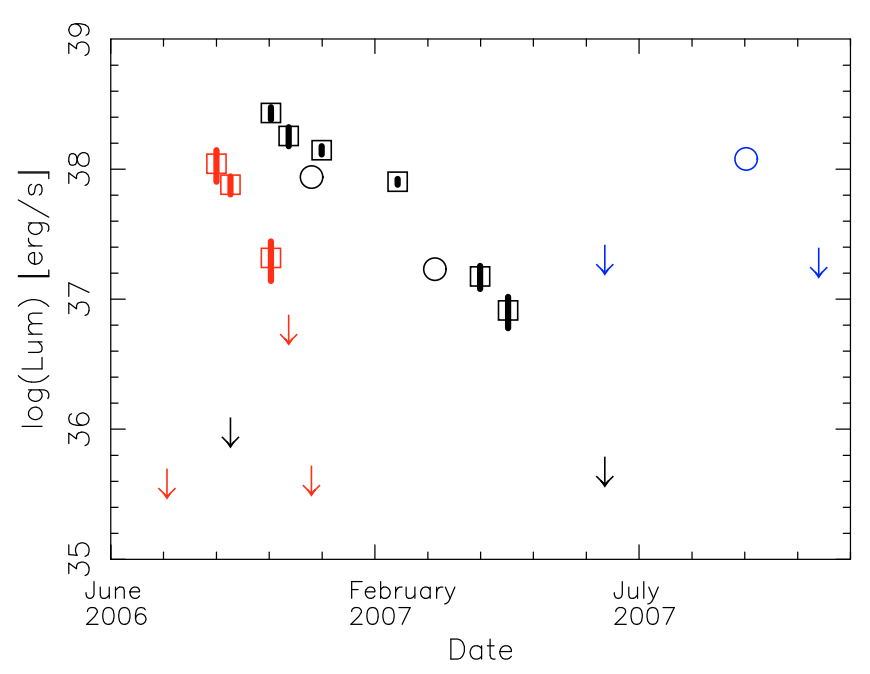

Fig. 2. The light curves of the three sources, XMMU J004215.8+411924 (red), SWIFT J004217.4+411532 (black), and M31N 2006-11a (blue). The arrows signify upper limits, the squares indicate data points where fits with spectral information were employed, and the circles correspond to luminosities estimated from spectra with model parameters fixed at expected values.

very soft and the three models fit the spectra equally well. For $\mathrm{X}$-ray binaries in outburst, such a soft spectrum is always found to be emission from an accretion disc, and we therefore use the disc black-body interpretation in the following analysis. For this model, all fits yielded neutral hydrogen columns consistent with just the Galactic foreground absorption, and the fits were repeated with the column density fixed to this value. Due to the lack of spectral information from Chandra HRC, we used fixed models (with the parameters given in parenthesis in Table 2) to convert the count rates to fluxes in observations 8 and 9 . Finally we used the same fixed models to place upper limits on the luminosity of the source in observations 4 and 13. The light-curve is displayed in Fig. 2.

After a fast rise to the maximum luminosity of $\sim 3 \times$ $10^{38} \mathrm{erg} \mathrm{s}^{-1}$, the source luminosity decayed exponentially to a quiescent level a factor of $>500$ lower than the peak luminosity, with an e-folding timescale $\tau_{\mathrm{e}} \sim 30-40$ days. According to 


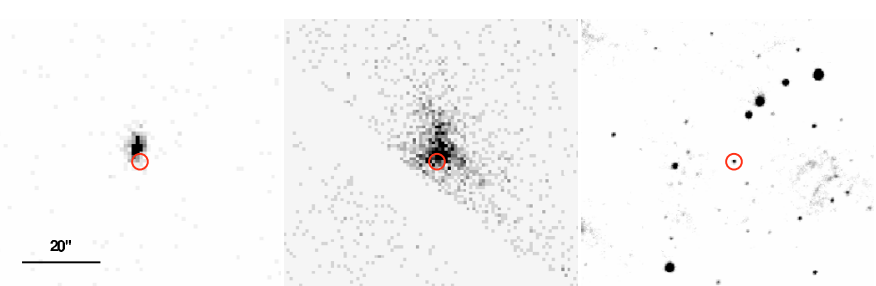

Fig. 3. The position of XMMU J004215.8+411924 in X-rays and optical. Observation 3 with Chandra ACIS-I (left), observation 4 with MOS2 (middle), and the $B$-band image from the LGS (right). The red circle has a radius of 2 arcsec and marks the previously identified optical counterpart to the $\mathrm{X}$-ray source.

the absorbed disc black-body fits the inner disc temperature decreased from $0.6 \mathrm{keV}$ at the peak luminosity to $\sim 0.25 \mathrm{keV}$. In Fig. 1 a comparison between the spectrum at the outburst peak and at the lowest detected luminosity is shown. The behaviour of the source is typical of black-hole candidate outbursts to the high/soft state (McClintock \& Remillard 2006).

We used observation 8 to find the most precise position of the source, $\mathrm{RA}(\mathrm{J} 2000)=00: 42: 17.3, \operatorname{Dec}(\mathrm{J} 2000)=+41: 15: 37.2$ ( $1 \sigma$ error $0.5 \operatorname{arcsec})$. We found no optical source at the X-ray position from searching the Swift UVOT observations, as well as from Galex and HST and the LGS observations. However, as for XMMU J004215.8+411924, the observations are not sensitive enough to constrain the source type.

\subsection{M31N 2006-11a}

This optical nova was detected by Itagaki on 2006 November 24, and it reached $17.3 \mathrm{mag}$ at maximum on unfiltered CCD images $^{3}$. The position was reported to be $\operatorname{RA}(\mathrm{J} 2000)=$ $00: 42: 56.81$, Dec $(J 2000)=+41: 06: 18.4$. From a spectral analysis of the optical nova, A. Shafter concluded that it was of the Fe II type ${ }^{4}$. We observed a transient source in only one observation (14), with Swift at the position of the nova. In this observation a soft X-ray source with about 26 counts is detected, just inside the field of view of the XRT, with all photons below $530 \mathrm{eV}$. The temperature of a black-body fit to the source spectrum can be constrained to $40 \mathrm{eV}<k T<90 \mathrm{eV}$, using the Galactic foreground absorption as a lower bound and constraining the luminosity to below $3.5 \times 10^{38} \mathrm{erg} \mathrm{s}^{-1}$ (the Eddington luminosity of a white dwarf at the Chandrasekhar mass limit, assuming an He-rich atmosphere).

Assuming a temperature of $50 \mathrm{eV}$ and the best-fit absorbing column of $1.1 \times 10^{21} \mathrm{~cm}^{-2}$, we derive upper limits to the luminosity before and after the detection. From observation 13 two months before the detection, we derive an upper limit of $2.0 \times 10^{37} \mathrm{erg} \mathrm{s}^{-1}$. From combining observations 15,16 and 17 we place an upper limit of $1.9 \times 10^{37} \mathrm{erg} \mathrm{s}^{-1}$ a month after the detection. The resulting lightcurve is shown in Fig. 2.

During observation 14, a Swift UVOT exposure of the nova region of $4.2 \mathrm{ks}$ in $U$ and $7.7 \mathrm{ks}$ in UVW2 was obtained. The nova was clearly detected in both filters at magnitudes $20.15 \pm 0.19$ and $21.12 \pm 0.16$, respectively. The emission in UVW2 $(180 \pm 20 \mathrm{~nm})$ may well result from the [CIII] $190.9 \mathrm{~nm}$, [NIII] $175.0 \mathrm{~nm}$ and/or [OIII] $166.3 \mathrm{~nm}$ lines, typical of the spectra of novae in the nebular phase.

\footnotetext{
${ }^{3}$ http://cfa-www.harvard.edu/iau/CBAT_M31.html\# $2006-11 \mathrm{a}$

${ }^{4}$ http://mintaka.sdsu.edu/faculty/shafter/ extragalactic_novae/HET/index.html
}

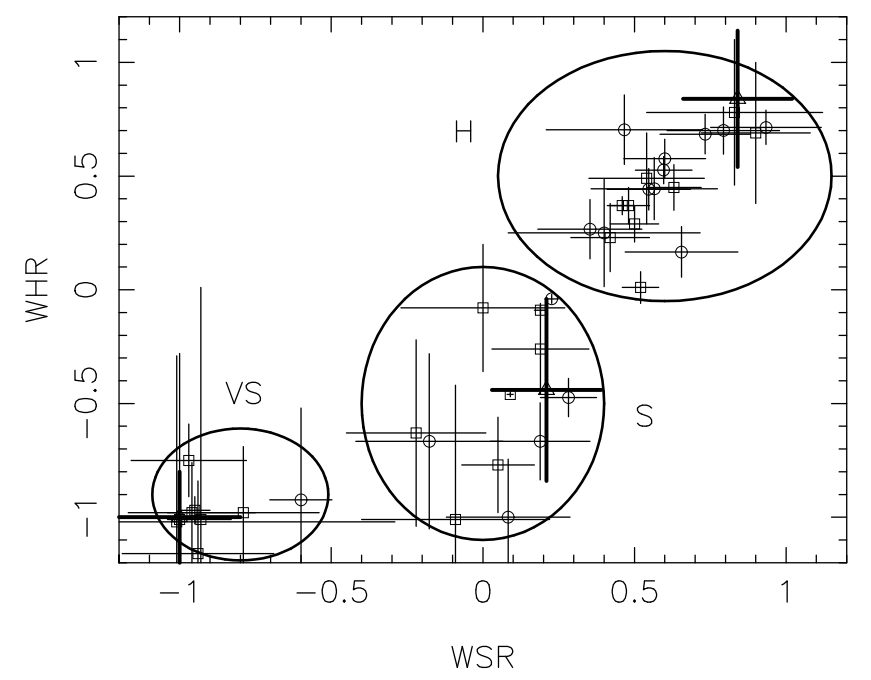

Fig. 4. The hardness ratios at the highest observed luminosities of 41 transients observed in M 31. The sources are divided into three subcategories: very soft (VS), soft (S) and hard (H). Open squares are data points from (Williams et al. 2006), while circles are data points from our analysis of transients listed in Voss \& Gilfanov (2007). The three sources analysed in this paper are marked with triangles and have thick error-bars. The displayed errors correspond to 1-sigma confidence intervals.

We searched for the optical source in observations before the detection. We detected the source neither in the Galex image nor in the existing HST observations, and the source was not inside the field of view of the UVOT in the Swift observations 5 and 6.

About 1.5 months after the X-ray detection, the source was detected in the UVW2 band in observations 15 and 17 with magnitudes of $21.95 \pm 0.30$ and $21.62 \pm 0.19$, respectively, and in the $U$-band in observation 16 with magnitude $20.63 \pm 0.11$, corresponding to a luminosity decrease of $\sim 0.5$ mag between observations 14 and 15 . The source was not detected in later observations.

While the X-ray luminosity dropped by a factor of 10 between observations 14 and 17 , the $U V$ luminosity only changes by a factor of 2 . This indicates that the strong decrease in the flux of observed X-rays is due to a decrease in the temperature of the emitting region. Assuming a temperature of $50 \mathrm{eV}$ for the source in observation 14 and an absorbing column of $1.1 \times 10^{21} \mathrm{~cm}^{-1}$, the upper limit on the X-ray to $U V$ ratio in observation 15 to 17 corresponds to a black-body temperature below $37 \mathrm{eV}$.

\section{Discussion}

To compare the sources discussed in this paper with previously observed sources and to understand how the fit into recognised classes of sources, we select a sample from the two catalogues of transient X-ray sources in M 31 (Williams et al. 2006; Voss \& Gilfanov 2007), based on the criteria that a variability of $>20$ is established and that spectral information must be available. This sample consists of 43 transients, observed over a period of 4.6 yr (October 1999-May 2004) in 45 Chandra observations, as well as with 5 epochs of XMM-Newton data. Because the spatial and temporal coverage is far from complete from these observations, we can set a lower limit to the rate of transients of $\sim 9$ transients $\mathrm{yr}^{-1}$, revising the lower limit of 6 transients $\mathrm{yr}^{-1}$ obtained by (Trudolyubov et al. 2006) somewhat upwards. 
We classified the sources, using the hardness ratios of the sources as given in Williams et al. (2006) $)^{5}$. We characterised the outburst by the hardness ratios at the highest observed luminosity. For the transients published in Williams et al. (2006), we used their values, whereas for the sources in Voss \& Gilfanov (2007), we analysed the Chandra observations to find the hardness ratios. As shown in Fig. 4, the sources can be naturally divided into three distinct populations (with the three sources discussed in this paper fitting into one class each): the very soft transients, the soft transients, and the hard transients. From this analysis we found that half (20) of the observed sources belong to the hard class, with the other half distributed evenly between the soft (12) and the very soft (9) sources.

\section{Conclusions}

For the first time, Swift has been used to observe X-ray transients in M 31. We analysed three sources, two of which were discovered from the Swift observations, thereby showing the possibilities of using Swift for such studies. We furthermore made use of all available Chandra and XMM-Newton observations to analyse the developments in luminosity and spectrum for each of the sources. We investigated the rate of transients in M 31 and found a minimum rate of $9 \mathrm{yr}^{-1}$, and we found that about half of the sources have a hard spectrum.

Acknowledgements. This research made use of data obtained from the Chandra Data Archive and software provided by the Chandra X-ray Center (CXC) in the application package CIAO and observations obtained with XMM-Newton, an ESA science mission with instruments and contributions directly funded by ESA Member States and NASA. The XMM-Newton project is supported by the Bundesministerium für Wirtschaft und Technologie/Deutsches Zentrum für Luft- und Raumfahrt (BMWI/DLR, FKZ 50 OX 0001), the Max-Planck Society. We thank the Swift team for their help with scheduling the TOO observations. We made use of observations made with the NASA/ESA Hubble Space Telescope and with the Galaxy Evolution Explorer, obtained from the data archive at the
Space Telescope Institute. STScI is operated by the association of Universities for Research in Astronomy, Inc. under the NASA contract NAS 5-26555. G.S. and H.S. are supported through DLR (FKZ 50 OR 0405).

\section{References}

Arnaud, K. A. 1996, Astronomical Data Analysis Software and Systems V, ed. G. Jacoby, \& J. Barnes, ASP Conf. Ser., 101, 17

Balucinska-Church, M., \& McCammon, D. 1992, ApJ, 400, 699

Campana, S., Colpi, M., Mereghetti, S., Stella, L., \& Tavani, M. 1998, A\&ARv, 8,279

Coe, M. J., Edge, W. R. T., Galache, J. L., \& McBride, V. A. 2005, MNRAS, 356 Garmany, C. D., \& Humphreys, R. M. 1985, AJ, 90, 2009

Haberl, F., Pietsch, W., Greiner, J., Ajello, M., \& the collaboration to monitor the supersoft state of optical novae in M 31 2006, ATel, 881

Han, C. 1996, ApJ, 472, 108

Kaaret, P. 2002, ApJ, 578, 114

Kong, A. K. H., Di Stefano, R., Garcia, M. R., \& Greiner, J. 2003, ApJ, 585, 298

MacDonald, J., Fujimoto, M. Y., \& Truran, J. W. 1985, ApJ, 294, 263

McClintock, J. E., \& Remillard, R. A. 2006, in Compact stellar X-ray sources, ed. W. Lewin, \& M. van der Klis (Cambridge, UK: Cambridge University Press)

Massey, P., Olsen, K. A. G., Hodge, P. W., et al. 2006, AJ, 131, 2478

Pietsch, W., Fliri, J., Freyberg, M. J., et al. 2005, A\&A, 442, 879

Pietsch, W., Haberl, F., Greiner, J., \& Stiele, H. 2006, ATel, 899

Pietsch, W., Greiner, J., Haberl, F., \& Sala, G. 2007a, Atel, 1116

Pietsch, W., Haberl, F., Sala, G., et al. 2007b, A\&A, 465, 375

Primini, F. A., Forman, W., \& Jones, C. 1993, ApJ, 410, 615

Skrutskie, M. F., Cutri, R. M., Stiening, R., et al. 2006, AJ, 131, 1163

Starrfield, S. 1989, in Classical Novae, ed. M. Bode, \& A. Evans (New York: Wiley), 39

Supper, R., Hasinger, G., Lewin, W. H. G., et al. 2001, A\&A, 373, 63

Tanaka, Y., \& Shibazaki, N. 1996, ARA\&A, 34, 607

Trinchieri, G., \& Fabbiano, G. 1991, ApJ, 382, 82

Trudolyubov, S. P., Borozdin, K. N., Priedhorsky, W. C., Mason, K. O., \& Cordova, F. A. 2002, ApJ, 571, 17

Trudolyubov, S., Priedhorsky, W., \& Cordova, F. 2006, ApJ, 645, 227

Voss, R., \& Gilfanov, M. 2007, A\&A, 468, 49

Wegner, W. 2000, MNRAS, 349, 193

Williams, B. F., Garcia, M. R., Kong, A. K. H., et al. 2004, ApJ, 609, 735

Williams, B. F., Naik, S., Garcia, M. R., \& Callanan, P. J. 2006, ApJ, 643, 356

Wilms, J., Allen, A., \& McCray, R. 2000, ApJ, 542, 914

Yan, M., Sadeghpour, H. R., \& Dalgarno, A. 1998, ApJ, 496, 1044

\footnotetext{
$5 \mathrm{WSR}=(\mathrm{WM}-\mathrm{WS}) /(\mathrm{WM}+\mathrm{WS})$ and $\mathrm{WHR}=(\mathrm{WH}-\mathrm{WS}) /(\mathrm{WH}+\mathrm{WS})$, where WS, WM and WH are the source counts in the $0.3-1.0,1.0-2.0$ and 2.0-7.0 keV band, respectively. Note that these ratios are different from the hardness ratios defined in Sect. 3.1
} 